\title{
Médiévales
}

Langues, Textes, Histoire

70 | printemps 2016

Lieux d'hygiène et lieux d'aisance en terre d'Islam (VII $\mathrm{e}_{-}$ $\mathrm{XV}^{\mathrm{e}}$ siècle)

\section{Les latrines en al-Andalus : leurs principales caractéristiques et les conditions sanitaires urbaines}

The Latrine in Al-Andalus : its Main Characteristics and the Urban Hygienic Conditions

\section{leva Reklaityte}

Traducteur : Sophie Gilotte

\section{(QpenEdition}

\section{Journals}

Édition électronique

URL : http://journals.openedition.org/medievales/7778

DOI : 10.4000/medievales.7778

ISSN : 1777-5892

\section{Éditeur}

Presses universitaires de Vincennes

Édition imprimée

Date de publication : 23 juin 2016

Pagination : 59-75

ISSN : 0751-2708

\section{Référence électronique}

leva Reklaityte, "Les latrines en al-Andalus : leurs principales caractéristiques et les conditions sanitaires urbaines », Médiévales [En ligne], 70 | printemps 2016, mis en ligne le 15 juin 2018, consulté le 19 avril 2019. URL : http://journals.openedition.org/medievales/7778; DOI : 10.4000/ medievales. 7778 
leva Reklaityte

\section{Les latrines en al-Andalus : leurs principales caractéristiques et les conditions sanitaires urbaines}

On ne peut concevoir la maison des villes d'al-Andalus sans une latrine ni un puits dans le patio. L'existence généralisée de latrines dans les demeures d'al-Andalus est un fait qui distingue la culture andalouse de celle qui régnait alors dans le reste de l'Europe. Cependant, il s'agit principalement d'une caractéristique urbaine, car les vestiges de l'habitat rural en ont gardé peu de trace. Comme la plupart des sociétés de l'islam médiéval, al-Andalus a développé une culture urbaine raffinée, ce dont témoignent les réseaux hydrauliques complexes et les solutions d'assainissements collectives, y compris pour les latrines des constructions domestiques. Peu connues par les textes et les images, car relevant de l'intime, c'est surtout par l'archéologie qu'une histoire des latrines peut aujourd'hui s'écrire. Les données textuelles nous renseignent plutôt sur des pratiques, difficiles à documenter archéologiquement, notamment lorsque l'accès aux latrines n'était pas possible ${ }^{1}$; dans ce cas, les cours d'eau, les jardins, voire les cimetières ou d'autres lieux peu fréquentés, pouvaient être utilisés en cas de nécessité.

Depuis les années 1980, les interventions archéologiques réalisées tant sur des établissements urbains dépeuplés que dans les villes qui conservent des vestiges de leur passé médiéval permettent de documenter et d'analyser les différentes solutions hydrauliques qui furent adoptées dans l'architecture domestique. Mais, indépendamment de la question de savoir si la latrine débouchait dans un puisard ou dans un égout, l'existence généralisée de latrines dans les demeures d'al-Andalus est un fait bien plus important. Elles permettent en effet de mieux comprendre l'insertion des latrines dans

1. E. García Gómez et É. LÉvi-Provençal, Sevilla a comienzos del siglo XII. El tratado de Ibn 'Abdūn, Madrid, 1992, p. 109 ; IBN AL-ATHĪR, Annales du Maghreb et de l'Espagne, éd. E. FAGNAN, Alger, 1898, p. 131-132. 
leur environnement hygiénique et, à travers celui-ci, les rapports à l'intime et la propreté qui pouvaient exister dans la société andalouse du Moyen Âge. La place des latrines dans les constructions domestiques se trouve ainsi à l'articulation de deux espaces que nous étudierons successivement : l'espace extérieur de la ville par leur rattachement - ou non - à des réseaux d'assainissement, collectifs ou privatifs, et l'espace intérieur de la maison.

\section{Les latrines et leur insertion dans les réseaux hydrauliques urbains}

\section{Les systèmes d'évacuation simples et traditionnels}

En règle générale, outre les cloaques, tous les cours d'eau - fleuves, ruisseaux et lits de torrents inclus - pouvaient représenter un moyen adéquat pour se débarrasser des eaux usées ou des déchets domestiques. De fait, l'emplacement de certaines latrines sur un point haut, donnant sur un précipice, permettait à leurs usagers de se passer d'un système d'assainissement puisque les résidus tombaient alors directement dans le vide. Ce moyen d'évacuation des eaux est documenté, entre autres lieux, dans les qașaba-s de Malaga, d'Almería, dans le quartier de la qașaba almohade de Paderne (Albufeira, Algarve portugaise) et même dans le complexe palatin de l'Alhambra (Grenade) ${ }^{2}$.

De fait, la latrine n'apparaît pas comme un élément indispensable dans les campagnes et, dans beaucoup d'établissements de caractère éminemment rural, on n'en détecte aucune, ni d'ailleurs d'autre système d'évacuation des eaux résiduelles. Ainsi, dans l'établissement rural de Las Sillas (Marcén, Huesca), fondé au $\mathrm{X}^{\mathrm{e}}$ siècle, on a pu documenter un bassin, des réservoirs d'eau, une hypothétique vasque à ablutions, des canalisations de largeur et profondeur diverses taillées dans la roche, dont certaines débouchent sur la falaise, profitant de la situation en hauteur du site ${ }^{3}$. En dépit de leur grand nombre, il n'est pas possible d'identifier ces canaux comme étant des déversoirs pour les eaux résiduelles et il est plus probable qu'ils aient été destinés à évacuer les eaux pluviales. Une situation similaire se retrouve à Vascos (Navalmoralejo, Tolède), une grande agglomération de caractère plutôt rural où, en dépit des évacuations prévues pour les eaux

2. M. J. SerRa Godinho CoElHo Belo Dos SANTOS, Águas do quotidiano. Estruturas habitacionais islâmicas no territorio algarvio (Tese para obtenção do grau de mestrado, Universidade do Algarve), Faro, 2013 ; I. REKLAITYTE, Vivir en una ciudad de al-Ándalus : hidráulica, saneamiento y condiciones de vida, Saragosse, 2012, p. 179-193.

3. P. SÉNAC, Un «village » d'al-Andalus aux alentours de l'an Mil. Las Sillas (Marcén, province de Huesca), Toulouse, 2009. 
des pluies, il ne semble pas avoir existé de système équivalent pour les eaux résiduelles ${ }^{4}$.

Dans le cas de l'habitat rural d'époque almohade d'Alcariais de Odeleite (Castro Marim, Algarve, Portugal), la conduite issue de la seule latrine documentée passe à travers un mur, sans que le puisard qui aurait dû lui être associé n'ait été découvert. Il est envisageable que d'autres latrines du site aient pu fonctionner de la même manière, c'est-à-dire en débouchant de l'autre côté d'un mur sans qu'il y ait pour autant de puits ou de fosse pour recevoir les eaux fécales ${ }^{5}$. Dans ce cas concret, Maria José Serra croit y déceler une certaine prudence hygiénique de la part des occupants de ces demeures, en arguant que l'exposition à l'air libre et à la vue des excréments n'aurait pas pour autant contribué à la contamination de l'environnement grâce à l'orientation septentrionale du secteur, qui les protégeait d'une exposition directe au soleil ${ }^{6}$. Néanmoins, il est malaisé de défendre cette hypothèse puisque les matières fécales auraient de toute façon contribué à polluer les rues. Finalement, nous pouvons mentionner l'établissement maritime de Poço Antigo (Cacela Velha, Algarve, Portugal), dans lequel plusieurs conduites ouvertes pour drainer des eaux pluviales ont pu être documentées ; selon la même auteure, il est possible que certaines d'entre elles aient servi à évacuer des eaux résiduelles ${ }^{7}$.

Dans les cités les plus importantes comme Cordoue ou Saragosse, le remploi de structures hydrauliques anciennes était un fait bien visible. Dans ces deux villes, si l'utilisation continue des égouts romains est patente, la perte progressive de leur maintenance est également visible. De plus, on observe dans le cas du quartier d'al-Rușāfa de Cordoue la réutilisation, au cours des dernières décennies du $\mathrm{X}^{\mathrm{e}}$ siècle, d'un tronçon de l'aqueduc antique - el Aqua Augusta - non plus pour acheminer l'eau potable, mais pour évacuer les eaux usées ${ }^{8}$. À Saragosse, l'apparition des puisards remonterait au XI ${ }^{\mathrm{e}}$ siècle : ils n'auraient apparemment pas été nécessaires auparavant en raison de l'emploi des cloaques antiques ${ }^{9}$. En outre, de nouvelles branches de canaux étaient venues se greffer à ce réseau antérieur. Toutefois, l'essor

4. R. IZQUIERDO BENITO, « La vivienda en la ciudad hispanomusulmana de Vascos (Toledo). Estudio arqueológico », dans A. BAZZANA et J. BERMúDEZ LóPEZ éd., La casa hispano-musulmana. Aportaciones de la arqueología, Grenade, 1990, p. 147-163 (p. 149).

5. M. J. Serra Godinho Coelho Belo Dos Santos, Águas do quotidiano..., p. 8082.

6. Ibid.

7. Ibid.

8. J. F. Murillo, F. Castillo, E. Castro, M. T. CASAl, T. Dortez, « Los arrabales del sector septentrional del Yanib al Garbi », dans D. VAQUERIzo et J. F. MurILLO éd., El Anfiteatro romano de Córdoba y su entorno urbano. Análisis arqueológico (ss. I-XIII d.C.), Cordoue, 2010, p. 609.

9. F. EsCUDERO EsCUDERO et M. P. GALVE IZQUIERDO, Las cloacas de Caesaraugusta y elementos de urbanismo y topografía de la ciudad antigua, Saragosse, 2013. 
de l'activité constructive à l'époque des taifas vint modifier la trame des rues, altérant par là même les infrastructures souterraines romaines. Vers le milieu du XI ${ }^{\mathrm{e}}$ siècle, les puisards perforèrent les égouts, marquant l'abandon du système d'assainissement hérité des Romains, tandis que celui-ci ne semble connaître aucune réparation ni agrandissement.

Certains sites conservent la trace de réformes ayant affecté les installations sanitaires, peut-être à des moments où la pression démographique se fit plus forte. À Lérida, à l'époque des taifas, ou dans le faubourg califal de «Casas del Naranjal » (Cordoue), le tracé des rues fut modifié avec l'introduction de conduites souterraines qui rendit caduc le système des puits perdus ${ }^{10}$. Ces travaux d'amélioration des structures d'assainissement doivent certainement être considérés comme émanant de la collectivité locale. Ainsi, dans certaines villes, comme celle de Murcie, il a été possible d'observer le réseau d'assainissement complexe qui permettait d'évacuer les eaux usées par des égouts collectifs, et de se passer de la sorte de puisards ${ }^{11}$. En revanche, les habitants d'autres localités (Siyāsa,

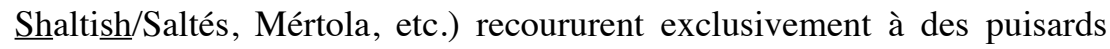
pour évacuer les eaux usées ${ }^{12}$.

\section{Les latrines et les voies publiques: le puisard}

Parmi les différentes solutions adoptées durant le Califat se trouve celle de latrines raccordées à des puisards, même s'il existait des égouts, principalement destinés à évacuer les eaux pluviales ${ }^{13}$. La rue et l'impasse

10. A. LorientePérez et A. Oliver Castaños, L'antic Portal de Magdalena, Lérida, 1992 (p. 109-111) ; C. CAMAChO CRuZ, M. Haro Torres, J. M. LARA Fuillerat, C. PÉrez NAVARRO, «Intervención arqueológica de urgencia en el arrabal hispanomusulmán "Casas del Naranjal”. Yacimiento “D”. Ronda Oeste de Córdoba », Anuario Arqueológico de Andalucía, 01 (2004), p. 210-230.

11. J. Navarro Palazón, P. JimÉnez Castillo, « El agua en la ciudad andalusí », dans J. Sobrino SimAl et L. CERVERA Pozo éd., El agua en el $2^{\circ}$ Coloquio internacional irrigación, energía y abastecimiento de agua: la cultura del agua en el arco mediterráneo, Alcalá de Guadaíra, 2010, p. 147-254 ; J. NAVARro PALAZÓn, P. JiMÉNEZ CASTILlO, « La gestión del agua en la ciudad andalusí: el caso de Murcia », dans J. M. GómEZ EsPín et R. M. HERvÁs AvilÉs éd., Patrimonio hidráulico y cultura del agua en el Mediterráneo, Madrid, 2012, p. 105-143.

12. A. BAZZANA, «Urbanismo e hidráulica (urbana y doméstica) en la ciudad almohade de Saltés (Huelva) », dans J. NAVARro PALAZÓn éd., Casas y palacios de al-Andalus, siglos XII-XIII, Barcelone/Madrid, 1995, p. 139-156 ; S. MACÍAS, Mértola islâmica. Estudo histórico-arqueológico do Bairro da Alcáçova (séculos XII-XIII), Mértola, 1996 ; J. NAVARRO PAlAZÓn et P. JimÉnez CASTILlo, Siyāsa. Estudio arqueológico del despoblado andalusí (ss. XI-XIII), Murcie, 2005.

13. Voir par exemple : L. APARICIO SÁNCHEZ, «Redes de abastecimiento y evacuación de aguas en los arrabales califales de Córdoba », Arte, arqueología e historia, 15 (2008), p. 237-256 ; E. CASTRO DEL RÍo, El arrabal de época califal de la zona arqueológica de Cercadilla : la arquitectura doméstica, Cordoue, 2005 ; B. VÁZQUEZ, « La gestión del agua 
de la ville médiévale se voyaient généralement attribuées des fonctions diverses, parmi lesquelles celle d'imposer l'implantation des structures d'assainissement. Les puisards et les égouts souterrains y étaient installés pour ne pas contaminer les espaces domestiques privés. Parallèlement, certains riverains évacuaient les eaux pluviales depuis leurs patios jusqu'à l'extérieur au moyen de gouttières constituées d'éléments céramiques emboîtés. De cette manière, réseau viaire et réseau d'assainissement étaient étroitement liés.

En al-Andalus, les puisards constituaient l'élément principal des installations sanitaires et il n'était pas rare de trouver des latrines reliées à ceux-ci même dans les villes où il existait un réseau partiel d'égout. Comme nous l'avons déjà signalé, Murcie sort du lot, puisqu'en raison de l'extension de son réseau d'assainissement collectif, ces puits noirs y faisaient figure d'exception ${ }^{14}$. Le cas des quartiers extra muros de Cordoue tranche également sur celui des autres localités d'al-Andalus en raison du rôle très important octroyé à l'eau dans leur configuration urbaine.

La présence de latrines dans quasiment toutes les demeures fouillées de ces vastes quartiers hors les murs de Cordoue a permis d'appréhender certaines caractéristiques constructives qui ne semblent pas fortuites ; ainsi, de petites canalisations en pierre, décrites par Belén Vázquez, sont très courantes dans les unités domestiques des faubourgs occidentaux ${ }^{15}$. Leur standardisation suggère qu'elles étaient produites en masse par des ateliers spécialisés dans la fabrication des structures hydrauliques domestiques (fig. 1a et 1b).

Nous connaissons d'autres madīnat-s (Dénia, Lérida, etc.) dont les maisons étaient presque toujours pourvues de latrines ${ }^{16}$. Il paraît évident que les habitants d'al-Andalus cherchèrent des solutions simples et pratiques pour résoudre les problèmes soulevés par l'assainissement domestique, comme le montrent l'usage de puisards, lorsque le terrain le permettait, ou la mise en place de formes différentes d'approvisionnement hydrique. Dans d'autres cas, les connaissances techniques et la prospérité économique donnèrent lieu à des solutions plus complexes, comme les

en los arrabales occidentales de Madinat Qurtuba », dans D. VAQUERIZO et J. F. MURILLO éd., El Anfiteatro romano de Córdoba..., p. 643-651.

14. J. NAVARro PALAZÓN, Una casa islámica en Murcia. Estudio de su ajuar (siglo XIII), Murcie, 1991.

15. B. VÁZQUEZ, « La gestión del agua... », p. 649.

16. J. A. Gisbert SANTOJA, « Dāniya y la villa de Denia. En torno al urbanismo de una ciudad medieval », dans R. AZUAR, S. GUTIÉRREZ, F. VALDÉS éd., Urbanismo medieval del País Valenciano, Madrid, 1993, p. 63-105 ; A. LORIENTEPÉREZ et A. Oliver CASTAÑos, L'antic Portal...; A. TORREMOCHA SILVA, « Abastecimiento y desalojo de aguas residuales en las ciudades andalusíes (siglos X-XV) », Estudios sobre patrimonio, cultura y ciencia medievales, 9-10 (2008), p. 225-273 entre autres. 

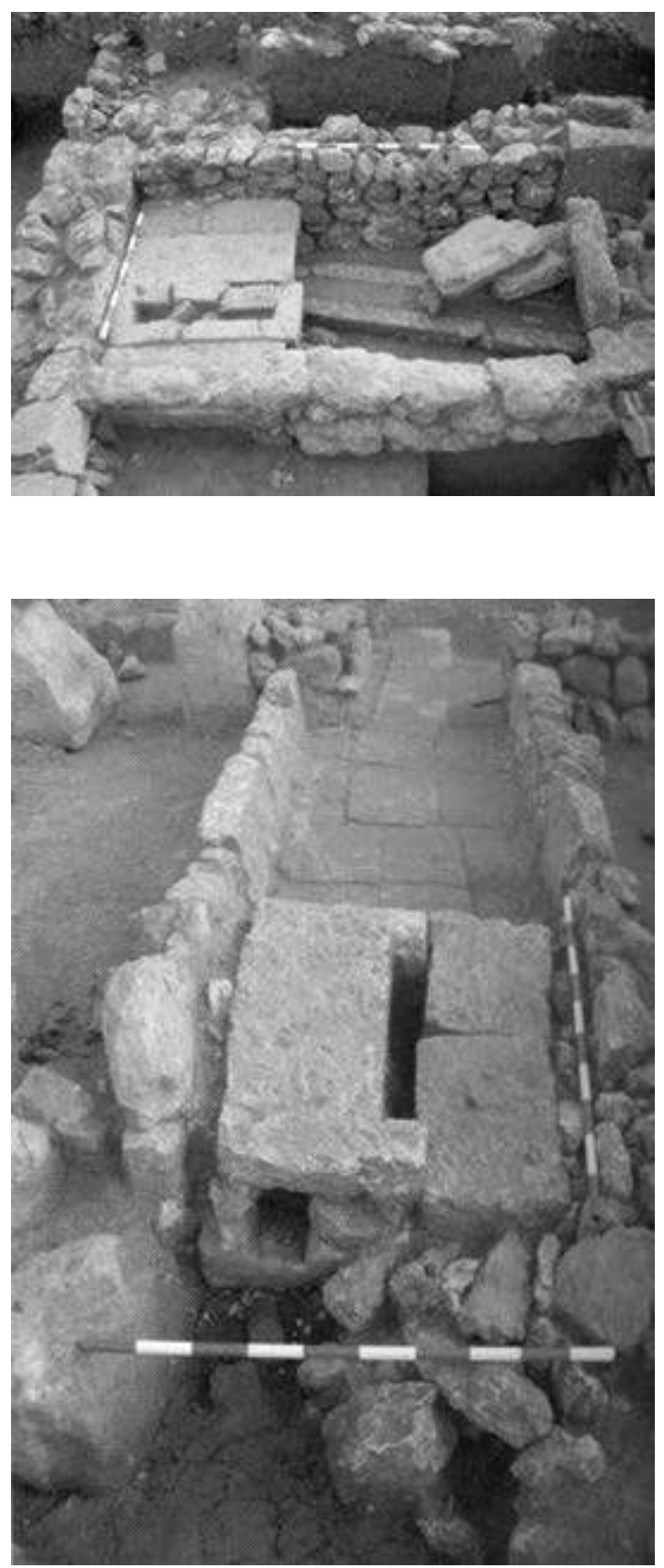

Fig. 1a. et fig. 1b.Latrines du faubourg califal de Trassierra (Cordoue)

(D'après S. RoDERo PÉREZ et J. A. MOLINA MAHEDERO,

« Un sector de la expansión occidental de la Córdoba islámica :

el arrabal de la carretera de Trassierra (I) », Romula, 5 (2006), p. 219-294 (p. 288)) 
réseaux d'égout, tout en cherchant toujours la meilleure solution pour se débarrasser rapidement et efficacement des résidus.

Dans le cas de Murcie, l'excédent d'eau des patios ou des puits servait à nettoyer la pièce d'aisance, en charriant les eaux noires jusqu'aux réseaux d'assainissement ${ }^{17}$. Toutefois, il n'était possible de faire converger l'expulsion des eaux pluviales et résiduelles par la latrine que si celle-ci était reliée au réseau d'assainissement collectif. En revanche, la conduite qui connectait la latrine au puisard était nécessairement séparée de celle qui évacuait l'eau du patio puisque, dans le cas contraire, celui-ci aurait pu déborder ${ }^{18}$.

Même si nous nous intéressons ici surtout à l'espace domestique et moins à la morphologie urbaine, il est important d'y faire référence puisque la localisation des puisards et leur proximité avec les maisons avaient des répercussions directes sur les conditions d'hygiène. Le curage et le nettoyage de leurs abords représentaient un travail plutôt désagréable et impliquaient une gêne tant par la pestilence qu'ils généraient que par la nécessité d'avoir recours à une main d'œuvre spécialisée. Les puisards étaient habituellement creusés dans les rues, afin de rendre plus facile cette maintenance tout en évitant la contamination de la zone résidentielle ou l'accumulation de mauvaises odeurs à l'intérieur des maisons.

La trame urbaine des faubourgs de Cordoue est très représentative de l'emploi des latrines et de leurs réceptacles : ces derniers y étaient creusés dans les rues, toujours à proximité du mur mitoyen des latrines. Les eaux fécales étaient acheminées vers les fosses à l'aide de canalisations en pierre, céramique ou briques, selon les cas ${ }^{19}$. Cette solution se retrouve, bien qu'à moindre échelle, dans les localités de caractère rural, du moins quand des latrines y existent.

D'autres villes d'al-Andalus présentaient également, au moins de façon partielle, cette configuration de latrines reliées à des puits perdus situés dans des impasses ou sur les voies publiques. Ces derniers étaient normalement couverts par des dalles de pierre légèrement nivelées par de la terre battue ${ }^{20}$. Il est intéressant de noter que, dans le cas des maisons almohades du Convento da Graça de Tavira (Faro, Algarve), au contraire, ils ont été systématiquement installés dans les impasses privées et au sein des espaces domestiques, mais jamais dans l'espace public ${ }^{21}$.

17. J. NAVArro Palazón et P. JimÉnez CAStillo, « El agua en la vivienda andalusí: abastecimiento, almacenamiento y evacuación », Verdolay, 7 (1995), p. 401-412.

18. Ibid.

19. C. Camacho Cruz, M. Haro Torres, J. M. Lara Fuillerat, C. Pérez Navarro, «Intervención arqueológica... », p. 210-230.

20. S. MACÍAS, Mértola islâmica..., p. 87.

21. M. J. Serra Godinho Coelho Belo Dos Santos, Águas do quotidiano..., p. 112. 
L'emplacement des puisards dans les rues répondait à la volonté de ne pas polluer et empuantir la maison : les traités de hisba se font l'écho de cette préoccupation pour l'hygiène collective, en interdisant d'en creuser s'ils ne se trouvent pas à proximité immédiate des demeures ; ainsi, dans la Séville almoravide, Ibn 'Abdūn précise que les puisatiers ne devaient pas forer dans les rues afin de ne pas gêner et salir les passants ${ }^{22}$.

Mais la réalité quotidienne était parfois très éloignée de ce que préconisaient ces traités et il n'est pas rare de trouver, tant dans des villes (madīnat-s) que des villages (qarya-s), des puits perdus déplacés, envahissant de façon plus ou moins marquée ces emplacements privilégiés que constituaient voies publiques et impasses ${ }^{23}$.

Quoique ce ne fût pas leur emplacement habituel, les puisards pouvaient parfois prendre place ailleurs, dans les maisons. Les habitants devaient alors vivre très près de leurs propres résidus, subissant les gênes que cela ne devait pas manquer d'occasionner. Les puisards pouvaient alors être creusés dans le patio ; ils en occupaient alors un angle ou un recoin, toujours à l'opposé du puits d'eau potable ${ }^{24}$. Un autre endroit était le vestibule, la latrine étant située directement au-dessus et sans doute séparée par un système de fermeture, comme cela a été documenté dans plusieurs sites $^{25}$. On signalera aussi le cas des puisards établis dans les étables, et ce tant en milieu urbain que dans des zones rurales (fig. 2).

Parfois l'absence de revêtement spécifique au sol et de séparation entre les deux zones indiquent d'ailleurs qu'il n'existait pas de différenciation entre la pièce de la latrine et l'étable ${ }^{26}$. Il est même possible, tout particulièrement en zones rurales, qu'il n'existât aucun aménagement spécifique (ni latrines, ni puisard) et que l'étable pût avoir vu s'accumuler les niveaux de stabulations tant animaux qu'humains.

Un fait relativement exceptionnel mérite d'être mentionné : la localisation du puisard dans une pièce exiguë spécifique. À Siyāsa et

22. E. García GómeZ et É. Lévi-Provençal, Sevilla ..., p. 155.

23. J. NAVARRo PALAZÓn et P. JimÉnEZ CASTILlo, Siyāsa..., p. 189.

24. I. REKLAITYTE, Vivir en una ciudad..., p. 46-48.

25. E. RUIZ NiETO, «Intervenciones arqueológicas en el Polígono de Poniente durante los años 1993 y 1994 », Anuario Arqueológico de Andalucía, 95 (1999), p. 104-112 ; M. D. Ruiz Lara, J. F. Murillo Redondo, M. Moreno Almenara, « Memoria de los trabajos arqueológicos efectuados por la G.M.U. en el "vial H" del Polígono 3 del Plan Parcial de Poniente y en la unidad de actuación P-6 del P.G.O.U. de Córdoba (1995-1997) », Anuario Arqueológico de Andalucía, 97 (2001), p. 148-162 ; I. REKLAITYTE, Vivir en una ciudad..., p. 51-52.

26. C. CAMACho CRUZ et M. Haro TORRES, «Intervención arqueológica de urgencia en yacimiento Electromecánica. Campaña 2004. Ronda oeste de Córdoba », Anuario Arqueológico de Andalucía, 04 (2009), p. 1082-1093. 


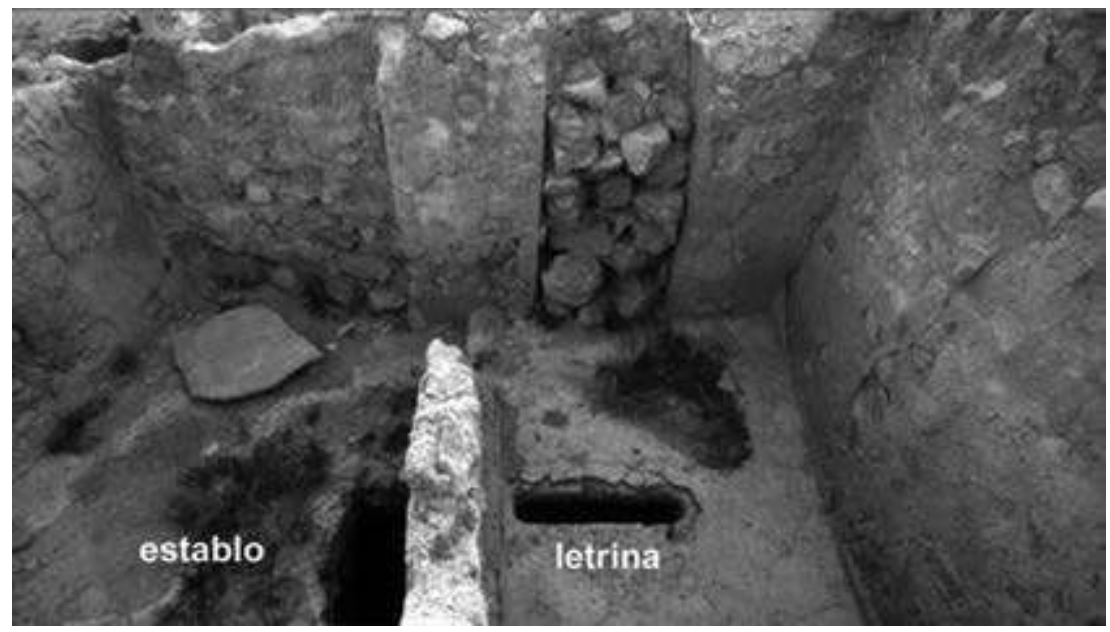

Fig. 2. Latrine avec puisard dans une étable de Siyāsa, Cieza

(D'après Yacimiento islámico de Siyāsa/Cieza (CD-ROM), Ayuntamiento de Cieza, sans date)

Cordoue, des cagibis situés entre la cour et la rue les abritaient ${ }^{27}$. Cela peut révéler, à notre avis, que l'utilisation des latrines était partagée entre voisins ; cela semble d'autant plus probable dans le cas de Siyāsa où le cabinet, sans communication avec la demeure, s'ouvrait directement sur l'impasse.

On soulignera, enfin, qu'aucun des deux facteurs, temporel et territorial, n'interféra à l'heure de creuser les puits noirs à l'intérieur de l'espace résidentiel. Le manque d'espace en fut, peut-être, la raison première et cela malgré les conséquences négatives de leur proximité telles qu'elles ont déjà été évoquées.

\section{La place des latrines dans l'architecture domestique : espace d'intimité}

L'emplacement des latrines à proximité de l'entrée des maisons, le plus près possible de la rue, répondait à une volonté de réduire la distance entre les latrines et le puisard ou l'égout (fig. 3 et fig. 4).

L'accès s'y faisait généralement depuis le patio ou le vestibule. Parfois, la surface réduite des maisons obligeait les propriétaires à

27. J. Navarro Palazón et P. JimÉnez Castillo, Siyāsa ..., p. 191-192 ; D. Botella ORTEGA, « Excavación arqueológica de urgencia en el Edificio Occidente (Parcela A-4, Manzana 9, Polígono 3, P1), de Córdoba », Anuario Arqueológico de Andalucía, 96 (2001), p. $143-152$. 


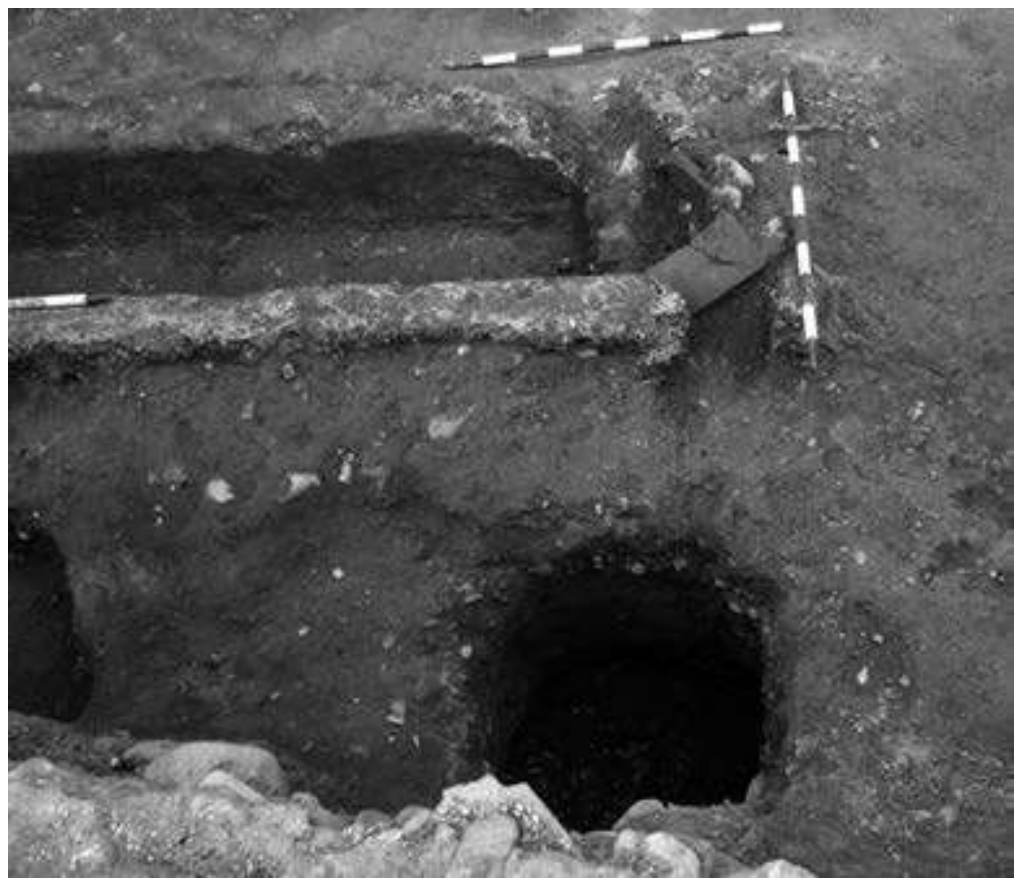

Fig. 3. Puisard et canalisation dans une maison califale de Saragosse (intervention archéologique dans la rue Cinco de Marzo)

(Cliché de F. J. Gutiérrez González)

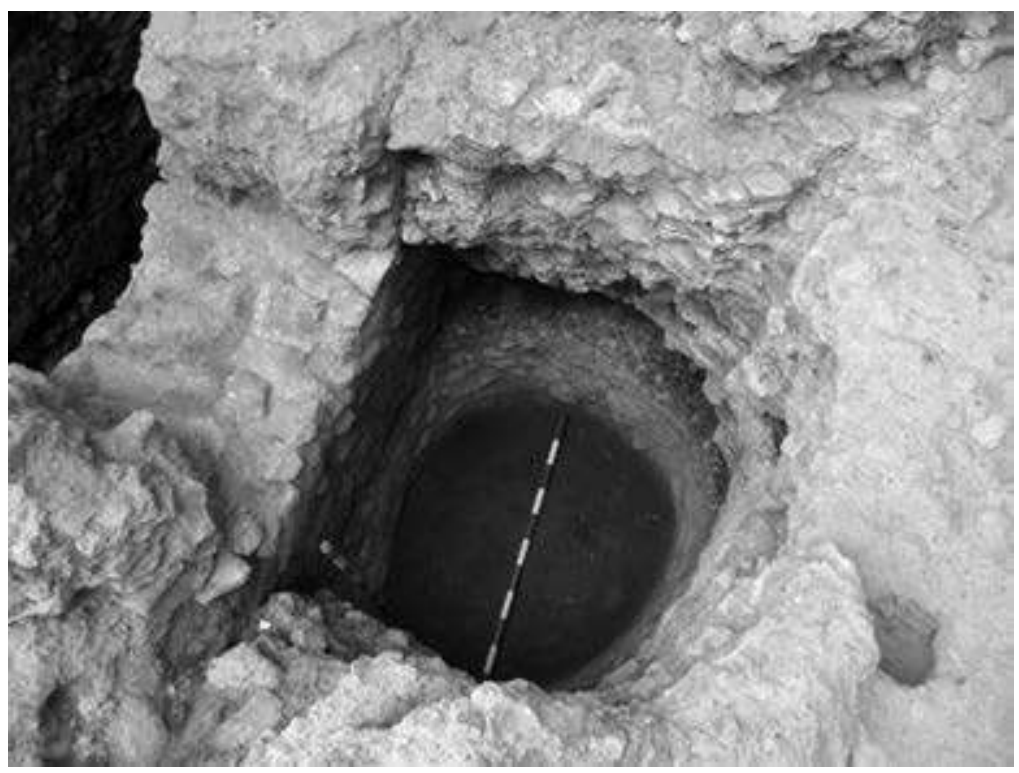

Fig. 4. Puisard installé sur la muraille romaine de Saragosse (Calle Mártires, 2-4) (Cliché de F. J. Gutiérrez González) 
chercher des alternatives ingénieuses, comme l'installation des toilettes sous la montée d'escalier. Ce type de solutions est documenté tant dans des demeures privées que dans des tours militaires (la Bāb al-Sharī'a, à l'Alhambra, par exemple) ou même dans les palais nasrides ${ }^{28}$. Le choix des lieux d'aisance favorisait ainsi non seulement l'expulsion rapide des résidus vers la rue - dans le puisard ou dans l'égout -, mais facilitait également sa ventilation. L'existence d'une ouverture, percée dans la partie haute de l'un des murs de cette pièce aux dimensions généralement très réduites, s'avérait nécessaire, tout en préservant l'intimité des usagers. Bien évidemment, les seuls témoins de ces petites fenêtres proviennent de sites où les édifices ont été conservés en élévation, comme c'est le cas à l'Alhambra de Grenade ; néanmoins, la plupart des latrines domestiques durent aussi disposer d'ouvertures semblables. Le patio jouait un rôle crucial : la plupart des latrines s'y ouvrant, il s'instaurait une circulation d'air entre celui-ci et les fenêtres, propice à l'aération ${ }^{29}$. L'air chaud étant moins dense que le froid et s'accumulant dans la partie supérieure des habitations, l'emplacement des ouvertures dans la partie haute des murs favorisait également le processus.

L'entrée des latrines devait être protégée des regards étrangers afin de sauvegarder l'intimité propre à ce type de lieux. Cette obligation se perçoit aussi bien dans l' architecture palatiale que dans l'habitat domestique. Un bon exemple nous est de nouveau fourni par les faubourgs califaux de Cordoue, où le recours à une entrée en coude permettait d'éviter que les usagers des latrines ne soient vus depuis le patio ${ }^{30}$. Une disposition semblable se retrouve au sein d'ensembles palatiaux, comme ceux de Madīnat al-Zahrā', ou de l'Alhambra ${ }^{31}$.

Différents matériaux et techniques étaient employés pour le sol des latrines, sans que le caractère utilitaire de cette pièce impliquât pour autant qu'ils fussent de qualité médiocre. Il est probable qu'en raison de la fonction de cet espace et malgré les soins que ne manquait sûrement pas d'apporter les occupants de la maison, il devait être difficile de le maintenir dans de bonnes conditions de propreté. En ce sens, la présence de surfaces dures devait considérablement aider aux tâches de nettoyage.

28. A. FernándeZ-Puertas, " La casa nazarí en la Alhambra », dans J. NAVARRo PALAZÓn éd., Casas y palacios de al-Andalus, Barcelone/Madrid, 1995, p. 269-287.

29. B. JIMÉNEZ ALCALÁ, «Aspectos bioclimáticos de la arquitectura hispanomusulmana », Cuadernos de la Alhambra, 35 (1999), p. 13-31.

30. C. Camacho Cruz, M. Haro Torres, J. M. Lara Fuillerat, C. Pérez Navarro, «Intervención arqueológica... »; E. CASTRO DEL Río, El arrabal..., p. 121.

31. A. Vallejo Triano, La ciudad califal de Madīnat al-Zahrā'. Arqueología de su excavación, Cordoue, 2010 (p. 255) ; A. FERNÁNDEZ-PUERTAS, The Alhambra from the Ninth Century to Yūsuf I (1354), Londres, 1997 (p. 64); J. BERMÚDEZ PAREJA, « El baño del Palacio de Comares, en la Alhambra de Granada. Disposición primitiva y alteraciones », Cuadernos de la Alhambra, 10-11 (1974-1975), p. 99-117. 
Indépendamment de la chronologie des bâtiments concernés, les matériaux de construction documentés sont très divers : depuis des pavements de pierre, incluant dallages de schiste, de grès vert ou même, dans des cas exceptionnels, de marbre, jusqu'à des sols de mortier de chaux ou de gravats, la terre battue étant, quant à elle, fréquente dans les zones rurales ou à l'intérieur de maisons modestes ${ }^{32}$. Mais les plus habituels sont généralement des pavements de calcaire ou de grès, des carreaux de céramique ou encore des briques.

Le dispositif des latrines est semblable dans toutes les demeures des villes d'al-Andalus : il consiste le plus souvent en un rehaussement du sol de quelques centimètres présentant un orifice dans sa partie supérieure, rainure exécutée avec plus ou moins de soin. Cette plateforme était construite en pierre, en brique et, de façon plus exceptionnelle, en marbre (comme c'est le cas à Madīnat al-Zahrā'). L'absence de planimétrie pour beaucoup de sites archéologiques fouillés interdit de connaître les dimensions de la plupart des latrines, même s'il est probable qu'elles variaient d'un site à l'autre : ainsi, à Madīnat al-Zahrā', la largeur du dispositif oscille entre 0,7 et $1,22 \mathrm{~m}^{33}$, tandis que dans les faubourgs cordouans elle mesure environ $0,60 \times 0,40 \mathrm{~m}$ avec une rainure de $0,35 \times 0,15 \mathrm{~m}^{34}$. Toutefois, la surélévation des latrines n'était pas systématique, de telle sorte qu'elles se trouvaient parfois au ras du sol comme cela est le cas, par exemple, à Siyāsa ${ }^{35}$.

Bien que les latrines soient considérées comme un espace privé purement fonctionnel, certaines d'entre elles présentent une décoration qui sort de ce que l'on attend habituellement dans un réduit ayant une telle fonction. Même si la plupart devaient être inconfortables et peu agréables - l'espace pour se mouvoir étant parfois inférieur à 0,60 $\mathrm{m}$ de large et $1 \mathrm{~m}$ de long ${ }^{36}$-, certaines personnes pouvaient se permettre le luxe de disposer de toilettes témoignant d'un certain souci esthétique. En dehors

32. Les demeures des faubourgs califaux de Cordoue présentent un très large éventail de revêtements de sols dans les latrines, depuis la terre battue jusqu'à des dalles de pierre. Toutefois, dans quelques sites, comme par exemple Saltés (Huelva) ou Silves (Faro), les latrines et les sols utilisent de manière quasiment systématique la brique. On trouvera des plus amples détails dans I. REKLAITYTE, Vivir en una ciudad..., p. 32-35.

33. A. VAllejo Triano, La ciudad..., p. 256.

34. J. F. Murillo Redondo, C. Fuertes SAntos, M. D. Luna OsunA, «Aproximación al análisis de los espacios domésticos en la Córdoba andalusí », dans F. GARCÍA VERDUGO et F. ACOSTA RAMíRez éd., Córdoba en la Historia. La construcción de la Urbe. Actas del congreso, Córdoba 20-23 de mayo 1997, Cordoue, 1999, p. 129-154.

35. J. NAVArro Palazón et P. JimÉnez Castillo, Siyāsa ..., p. 229-232.

36. A. Robles FERnÁNDEZ et E. NAVARRo SANTA-CRUZ, « Arquitectura doméstica andalusí y alfarería mudéjar en el arrabal de la Arrixaca. Memoria de la intervención realizada en un solar de la plaza Yesqueros-calle Toro (Murcia) », Memorias de Arqueología, 9 (1999), p. 572-600 ; A. JiMÉNEZ SANCHO, «Excavación en el Patio de los Naranjos de la Catedral de Sevilla. Una Mezquita amurallada », Anuario Arqueológico de Andalucía, 2000, III-3 (2003), p. 905-922. 
des résidences palatiales de Madīnat al-Zahrā' ou de l'Alhambra ${ }^{37}$, les exemples d'installations sanitaires recevant un décor sont très rares en al-Andalus. Cependant, le recours à des procédés décoratifs comme des peintures murales ou des pavements particulièrement soignés est attesté dans certaines propriétés d'individus aisés. On trouve, dans les faubourgs califaux de Cordoue et dans la qașaba de Silves (Faro, Algarve, Portugal), des latrines dallées de pierre de couleur inhabituelle, comme le calcaire violacé dans le cas cordouan ou le grès rouge, dans le cas lusitanien, dont la tonalité devait trancher sur celle des parois de la pièce, probablement enduites de chaux ${ }^{38}$. En dehors du cas particulier des édifices de caractère palatial qui viennent d'être cités, quelques résidences de riches propriétaires disposèrent de latrines aussi soignées que le reste des autres pièces : plusieurs demeures de Séville ou encore les résidences almohades du Palais d'Orive, à Cordoue, dotées d'une décoration de peinture murale en témoignent ${ }^{39}$.

L'eau ne pouvait faire défaut dans les installations sanitaires. Parfois, cependant, il existait des puits creusés à proximité des latrines et dont l'eau servait à leur nettoyage (comme sans doute dans les faubourgs de Cordoue), même si leur proximité avec les installations sanitaires rendait probablement leur eau impropre à la consommation humaine et la condamnait sans doute à n'être utilisée que pour des tâches de nettoyage ou pour les ablutions corporelles. Cette caractéristique est mise en évidence dans la ville de Murcie où les puits sont habituellement proches des latrines ${ }^{40}$. En outre, les apports d'eau ne devaient pas être trop importants sous peine de faire déborder le puits perdu.

La découverte de vasques en pierre de faible profondeur, destinées à contenir l'eau nécessaire à la toilette intime, n'est donc pas surprenante dans ce type de pièce, même s'il est possible que, dans la plupart des cas, on ait utilisé de simples récipients en céramique, comme ceux trouvés dans une des latrines du quartier de la qașaba de Mértola (Alentejo, Portugal) ${ }^{41}$.

37. A. FERnÁNDEZ-Puertas, « La casa nazarí... », p. 274 ; L. TORRES Balbás, «Letrinas y bacines », Al-Andalus, 24 (1959), p. 221-234. Sur les caractéristiques décoratives des latrines de Madīnat al-Zahrā', voir la contribution d'Antonio Vallejo dans le présent numéro de Médiévales.

38. E. CASTro Del Río, El arrabal..., p. 42 ; R. VARela Gomes, Silves (Xelb), uma cidade do Gharb Al-Andalus: a Alcáçova, Lisbonne, 2003, p. 92-94.

39. R. OJeda CALvo, «El edificio almohade bajo la casa de Miguel de Mañara », dans M. VAlor PIECHOTA et A. TAHIRI éd., Sevilla Almohade, Séville-Rabat, 1999, p. 135-141 ; A. CÁNOVAS UBERA et S. CARMONA BERENGUER, «El conjunto de pinturas tardoislámicas del Palacio de Orive », dans D. VAQUERIZO GIL éd., Guía arqueológica de Córdoba, Cordoue, 2003, p. 187.

40. J. Navarro Palazón et P. JimÉnez CAStillo, « El agua... », p. 401-412.

41. S. MACÍAS et C. TORRES, «El barrio almohade de la alcazaba de Mértola: el espacio cocina », dans J. NAVARRo PALAZÓN éd., Casas y palacios de al-Andalus, Barcelone/Madrid, 1995, p. 165-177. 
Dans le complexe palatial (qașr) de Madīnat al-Zahrā', la présence de telles vasques, alimentées en eau courante au moyen d'une canalisation semble réservée aux latrines de l'élite ${ }^{42}$. Dans d'autres cités d'al-Andalus, on en trouve faites de matériaux divers, comme dans certaines latrines de la ville émirale de Badjdjāna (Pechina, Almería), où elles sont en pierre ou en marbre, reliées à des conduites traversant les murs ${ }^{43}$. En résumé, différents matériaux servirent à leur fabrication : depuis le marbre, présent dans les complexes palatiaux, jusqu'à d'humbles récipients de céramique. D'une façon générale, elles peuvent avoir eu des fonctions multiples, et pour certaines seulement celle de bidets. Il faut signaler, enfin, que certaines cuves de bonne facture, surtout celles en marbre, ont pu faire l'objet de spoliation.

Même si les vasques trouvées dans les latrines pouvaient servir à la toilette des parties intimes, les ablutions rituelles s'effectuaient, au contraire, dans le patio ou dans les espaces domestiques destinés à de telles fins, selon ce que l'on peut apprécier dans certains établissements comme Siyāsa ${ }^{44}$. Dans ce gros bourg, des réduits, de faibles dimensions et ouverts sur le patio, étaient pourvus de plusieurs objets de céramique - une jarre et son support ainsi qu'une cuvette ayant pu servir à ces ablutions. D'hypothétiques réduits réservés à ces purifications ont été documentés dans d'autres sites archéologiques ${ }^{45}$. Leur utilisation est encore attestée par les Morisques ${ }^{46}$. Les cuvettes ainsi utilisées pour ces pratiques en contexte domestique pouvaient recevoir un dense décor estampillé incluant parfois même des éléments épigraphiques ; d'autres, plus simples, ont été documentées dans tout le territoire d'al-Andalus durant l'époque almohade ${ }^{47}$.

42. S. LóPeZ-Cuervo, Medina Az-Zahra: ingeniería y formas, Madrid, 1985, p. 35 ; et surtout A. VALLEJO TRIANO, La ciudad..., p. 258-260.

43. F. Castillo Galdeano, R. Martínez Madrid, M. Acién Almansa, « Urbanismo e industria en Baŷŷāna. Pechina (Almería) », dans II Congreso de Arqueología Medieval española (Madrid, 1987), Madrid, 1987, t. 2, p. 540-548.

44. J. NAVARro PALAZÓN et P. JimÉnez CASTillo, Siyāsa ..., p. 224-229.

45. Une possible pièce d'ablution a été découverte à Huerta Rufino (Ceuta) : J. M. HITA RUíz et F. VILLADA PAREDES, Un aspecto de la sociedad ceutí en el siglo XIV: los espacios domésticos, Ceuta, 2000, p. 39.

46. En 1608, la riche morisque aragonaise Esperanza Granada fut accusée par le tribunal d'Inquisition de disposer dans sa maison d'une pièce exiguë « [aux murs] enduits et [au sol] pavé de brique, qui indiquait qu'elle servait à faire le guadoc [les petites ablutions] et la zala [la prière] » (M. C. ANSÓN CALVO, Torrellas. Del esplandor morisco a la decadencia y la tendencia a su recuperación, Torrellas, 2014, p. 240).

47. F. CAVILla SÁnCheZ-Molero, La cerámica almohade de la isla de Cádiz (Ŷazīrat Qādis), Cadix, 2005, p. 288. Voir aussi la contribution de Claire Déléry dans le présent numéro de Médiévales. 
Pour conclure, rappelons tout d'abord que l'étude des conditions hygiéniques en al-Andalus en est à ses prémices. De nombreuses données font encore défaut pour prétendre restituer avec exactitude les conditions de vie des habitants d'al-Andalus. Celles-ci devaient, par ailleurs, être variables d'un contexte urbain à l'autre. Mais l'absence d'installations sanitaires peut parfois s'expliquer par des aménagements très sommaires - simples fosses peut-être surmontées de structures légères, en matériaux organiques -, qui n'auraient pas été reconnues lors des fouilles. Soulignons aussi que la découverte de canalisations souterraines n'implique pas en soi un haut niveau d'hygiène, puisqu'elles ont pu servir à drainer les eaux de pluie et non les eaux usées. De même, il est rare de détecter la présence d'égouts dans toute une ville, ceux-ci se concentrant dans certains quartiers, sans que disparaissent pour autant les puits perdus (fig. 5).

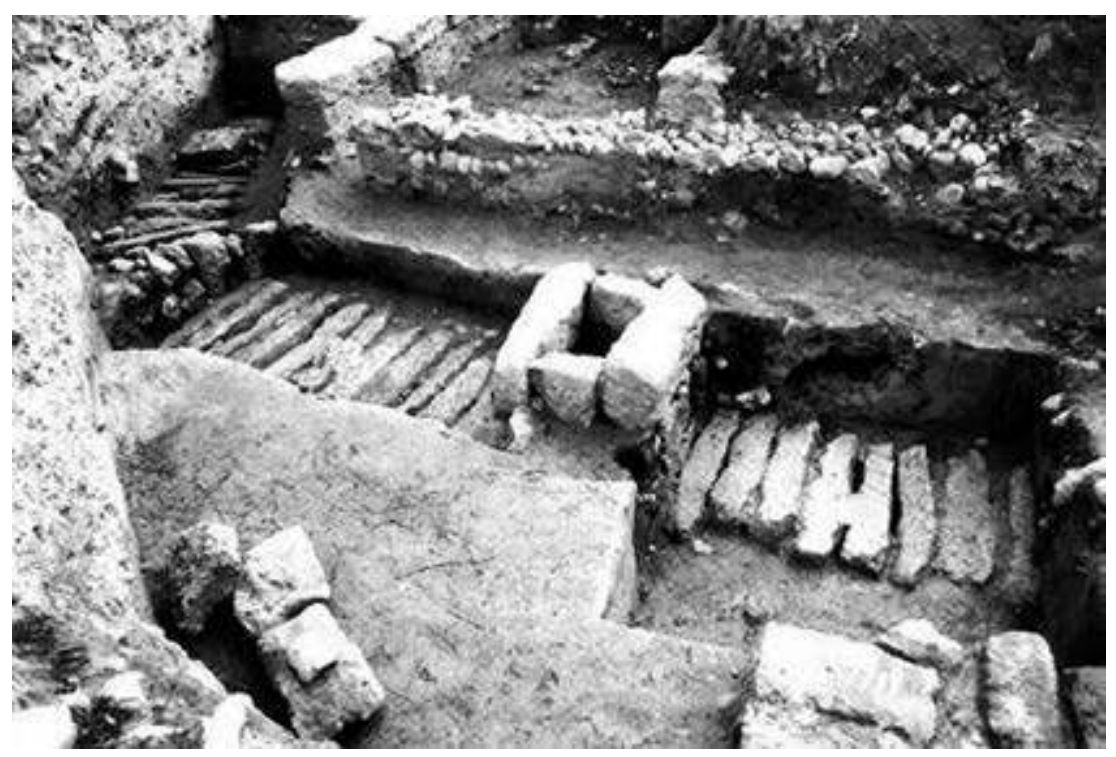

Fig. 5. Égout califal dans le zoo municipal de Cordoue

(D'après S. SÁNCHEZ MADRID, «Intervención arqueológica de urgencia en el Zoológico Municipal de Córdoba (tercera fase) », Anuario Arqueológico de Andalucía, 04 (2009), p. $1008-1022$ (p. 1021))

Finalement, on ne peut ignorer les aspects négatifs qu'impliquait l'installation de la latrine, tout spécialement la pollution ambiante, surtout lors de son nettoyage, la détérioration des murs de la maison comme conséquence de l'humidité, la dégradation de la voirie, la contamination des eaux ou la présence de mouches et autres vermines, les mauvaises 
odeurs, etc. Dans le cas du quartier almohade de la qașaba de Mértola, les archéologues ont pointé brièvement que les latrines associées aux puisards pouvaient avoir occasionné certains désagréments aux habitants du quartier ${ }^{48}$. Ces nuisances devaient être probablement plus accentuées durant les mois les plus chauds et dans les quartiers les plus densément peuplés, tandis que la situation différait vraisemblablement dans les zones intramuros où des espaces non édifiés étaient disponibles - qu'il se soit agi de jardins ou même de terres cultivables (terrains agricoles).

Pour terminer, nous rappelons que la fréquentation des latrines est un acte intime. Leur emplacement dans l'architecture d'al-Andalus devait donc être mis en relation avec la recherche d'intimité qui caractérise l'architecture domestique islamique ${ }^{49}$. Par ailleurs, la volonté d'éloigner le réduit de la latrine était motivée non seulement par le besoin impératif de cette intimité mais également par celui d'échapper aux nuisances découlant de la fonction du lieu.

Texte traduit de l'espagnol par Sophie Gilotte

leva Reklaityte - URBS, Saragosse

\section{Les latrines en al-Andalus : leurs principales caractéristiques et les conditions sanitaires urbaines}

Les données archéologiques montrent combien l'intimité à l'intérieur des latrines était un aspect clé de la maison d'al-Andalus. Outre des rideaux ou des portes, le cabinet était souvent occulté par une cloison qui créait une entrée coudée et empêchait de voir, depuis l'extérieur, l'usager qui s'y trouvait. Ce réduit, habituellement très exigu, présentait un dispositif souvent légèrement surhaussé et de dimensions variables, fait en brique ou en pierre, et plus exceptionnellement en marbre, avec une fente étroite en son centre. Une petite ouverture était sûrement percée en haut des murs pour en assurer l'aération, elle-même facilitée par la circulation de l'air entre le patio et la rue. L'accès aux latrines s'effectuait généralement depuis le patio ou le vestibule. Même si certaines villes comme Murcie, par exemple, possédaient un ample réseau d'égouts, la solution la plus fréquemment adoptée pour l'évacuation était celle de puits perdus, tel que cela a été documenté dans de nombreuses agglomérations urbaines ainsi que dans la plupart des faubourgs califaux de Cordoue et dans des établissements de moyenne importance comme Siyāsa (Cieza, Murcie) ou Shaltish/Saltés (Huelva).

Al-Andalus - égouts - gestion de l'eau - latrines - puisards

48. S. Gómez, R. Lígia, S. Macias, « Habitat e utensilios na Mértola almóada », Cuadernos de Madīnat al-Zahrā', 7 (2010), p. 175-195 (p. 178).

49. H. MorTADA, Traditional Islamic Principles of Built Environment, New York, 2003. 


\section{The Latrine in Al-Andalus : its Main Characteristics and the Urban} Hygienic Conditions

As it can be observed through the archaeological data, in an Andalusi house the privacy and the intimacy were key aspects whilst the latrine was being used. Apart from curtains and doors, the privacy was often concealed by a low wall that created a bend entrance and impeded viewing the person that was using the toilet by anyone who would have approached the room. Normally a latrine itself was an extremely reduced space. A small rectangular platform of dissimilar dimensions made of brick or stone, exceptionally of marble, with a narrow opening in the middle was placed in there. Most likely slim apertures in the upper part of the wall were used to ventilate the room. Due to the orifice in the wall, the air would circulate freely from the patio to the street. The access to the latrine was usually performed from the patio or the hallway. Although some Andalusi towns, as Murcia, for example, presented an extensive sewer network system, the most common solution for sewage was a use of cesspits as it has been documented in the major part of the Caliphal suburbs of Cordoba, in the settlements such as Siyāsa (Cieza, Murcia) or Shaltish/Saltés (Huelva) among others.

Al-Andalus - Cesspits - Latrine - Sewers - Water Management 
